\title{
MedienPädagogik
}

Zeitschrift für Theorie und Praxis der Medienbildung

Themenheft Nr. 29: Die Konstitution der Medienpädagogik. Zwischen interdisziplinärem Forschungsfeld und bildungswissenschaftlicher (Sub-) Disziplin.

Hrsg. v. Christian Swertz, Wolfgang B. Ruge, Alexander Schmölz und Alessandro Barberi.

\section{Überlegungen zur Konstitution der Medienpädagogik}

Norbert Meder

\begin{abstract}
Zusammenfassung
In diesem Beitrag werden auf mehreren Ebenen notwendige Voraussetzungen und Konstitutionsbedingungen der Medienpädagogik vorgeschlagen. Dabei werden unter anderem die anthropologische von der erkenntnistheoretischen sowie der sozial- bzw. kulturwissenschaftlichen Grundlegung unterschieden, um aus unterschiedlichen Perspektiven deutlich zu machen, worin das Gemeinsame solcher Grundlegungen besteht, da sie sich durchwegs auf die «Bildsamkeit im Sinne der Bildung beziehen lassen. In diesem Zusammenhang wird deutlich, dass der pädagogische Handlungszusammenhang nicht determiniert, sondern offen ist. In einem zweiten Schritt wird dann auf die Medialität von Bildungsprozessen fokussiert. Kommunikations-, Darstellungs- und Interaktionsmedien werden unterschieden. In dieser pluralen Medialität wird abschliessend ein notwendiges Konstituens Allgemeiner Pädagogik und das Alleinstellungsmerkmal der Medienpädagogik ausgemacht.
\end{abstract}

Reflections on the constitution of media education

\begin{abstract}
In this paper constitutional conditions of media education on several levels are analysed. The anthropological perspective is distinguished from the epistemological point of view. Sociological and cultural aspects are reflected in order to show the common ground of such foundations of media education. It is pointed out that they converge in the german term «Bildsamkeit» (educability), which is directly connected to «Bildung» (education). In this context, it becomes clear that the pedagogical action is not determined, but is open. In a second step, the mediality of educational processes are focused and communication, presentation and interaction media a differentiated. In this plural mediality, a necessary constituent of general and media pedagogy is finally proposed.
\end{abstract}

\section{Einleitung}

Konstitution heisst in der Wortbedeutung zu allererst Zusammenstellung (lat: con = zusammen und stituere = stellen, vgl. Hogrebe 1971). Schon im Lateinischen konnte man eine horizontale und eine vertikale Zusammenstellung unterscheiden. Die 
horizontale Konstitution kann als lose Sammlung von Wissensbeständen oder als deren ordnende Zusammenstellung gefasst werden. Letzteres tut z. B. eine gute Medienkunde. Es kann sich dabei aber auch um die Zusammenstellung gesellschaftlicher und politischer Probleme handeln, die dazu geführt haben, sich mit Medienpädagogik zu beschäftigen. Schliesslich kann man auch die Fakten, Gründe und Ursachen der Entstehungsgeschichte der Disziplin «Medienpädagogik`zusammenstellen. Im logischen Sinne kann man die Zusammenstellung der Gründe horizontal verstehen, im zeitlichen Sinne aber auch vertikal, nämlich diachron. Man darf nur die zeitliche Genesis nicht mit dem logischen Aufbau verwechseln.

Die vertikale Zusammenstellung ist stets eher logisch verstanden worden. Man stellte die Gründe zusammen, die eine Erkenntnis oder eine Wissenschaft rechtfertigen. Das kann auf zwei Weisen geschehen. Einmal kann man von der Erkenntnis ausgehen und nach ihren logischen Bedingungen zurückfragen. Das ist die rekursive oder auch analytische Methode, die gleichsam von oben nach unten zu den impliziten Grundlagen fortschreitet. Eines der besten Beispiele ist Kants transzendentale Argumentation, die Bedingungen der Möglichkeit der Erkenntnis eines Gegenstandes ausfindig zu machen. Das zweite vertikale Vorgehen schreitet von unten, von der Grundlegung, nach oben zu dem, was grundgelegt werden soll. Das ist die konstruktive oder auch synthetische Methode, die zumeist als die eigentliche Grundlegung, Konstitution einer Erkenntnis oder einer ganzen Wissenschaft angesehen wird. Das ausgezeichnete Beispiel hierfür ist die axiomatische Mathematik. Sie legt die Grundlagen einer Theorie als eine möglichst geringe Zahl von Axiomen fest. Axiome sind Aussagen über den Gegenstandsbereich einer Theorie, die zwar nicht bewiesen werden können, aber für die Theorie unerlässlich sind.

Beide vertikalen Konstitutionen sind in sich noch einmal gedoppelt: Sie gehen auf das Was-Sein des Gegenstands der Theorie sowie auf die Methode, wie in der Theorie vorzugehen ist. So ist beispielsweise in der Theorie der natürlichen Zahlen ${ }^{1}$ das Axiom ‘ 1 ist eine Zahl e eine Konstitution der Sache nach. Das Axiom von der sogenannten vollständigen Induktion ${ }^{2}$ ist dem gegenüber die Konstitution der Methode, d. h. hier der Beweisführung im Gegenstandsbereich der natürlichen Zahlen.

Die Antike und vor allem die Spätantike ${ }^{3}$ haben sich sehr intensiv mit der Begriffsbildung beschäftigt. Die Frage ist, wie werden Begriffe gebildet und was bestimmt ihren Inhalt bzw. das Wesen der Sache, die durch den Begriff gefasst wird. Gehen wir beispielsweise von der obersten Gattung, dem obersten und abstraktesten Begriff, der alles umfasst, was ist, dem Begriff des Seienden aus, dann suche man eine Differenz, die diese oberste Gattung vollständig in zwei Begriffe (zwei Bereiche)

1 Natürliche Zahlen sind 1, 2, 3 usw.

2 Das Axiom lautet: Wenn eine Eigenschaft für die 1 gilt und wenn die Eigenschaft von einer Zahl auf ihren Nachfolger vererbt wird, dann gilt die Eigenschaft für alle Zahlen.

3 In der Theorie der arbor porphyriana, des Begriffsbaumes des Porphyrios. 
aufgliedert. Das tut beispielsweise die Differenz von sterblich und unsterblich. Diese Differenz ist methodisch, insoweit sie den Ausgangsbegriff einteilt und in zwei Arten gliedert. Sie konstituiert divisiv (teilend) - noch genauer: in Zwei-Einteilung (Dichotomie) - sterbliches Seiendes (Natürliches) und unsterbliches Seiendes (Göttliches). Damit wird zugleich auch das Wesen einer Sache festgeschrieben: Sterblichkeit für alles in der Natur, Unsterblichkeit für alles im Bereich des Göttlichen. Geht man noch einen Schritt weiter und unterteilt die beiden Arten entlang der divisiven Unterscheidung von vernünftig/unvernünftig, dann ist die Sache Mensch in ihrem Was-Sein als sterblich und vernünftig konstituiert, Gott als unsterblich und vernünftig, alles nichtmenschliche Natürliche als sterblich und unvernünftig und der Dämon als unsterblich und unvernünftig ${ }^{4}$. Diese Art der Begriffsbildung ist vielen heute nicht mehr klar. Sie ist aber immer noch im Gebrauch, wie man an der Zweieinteilung aller natürlichen Zahlen in gerade und ungerade Zahlen sieht. Die spezifische Differenz ist hier «durch-2-teibar) und «nicht-durch-2-teilbar).

Dieses klassische Beispiel sollte zeigen, dass bei der Grundlegung einer Wissenschaft oder auch nur einer Theorie stets die Sache, der Gegenstandsbereich, und die Methode grundgelegt werden müssen. Die Sache wird bei der Begriffsbildung durch konstitutive Differenzen definiert, und die Methode vollzieht sich in divisiven Differenzen. Die Unterscheidung, dass Konstitution den Gegenstandsbereich und Division die Methode betrifft, bildet den philosophiegeschichtlichen Hintergrund für die spätere Unterscheidung von konstitutiv und regulativ bei Kant.

Ich will im Folgenden die Grundzüge einer vertikal konstruktiven Konstitution der Medienpädagogik skizzieren. Als Kantianer hätte ich auch eine transzendentallogische vertikal-rekursive Konstitution wählen können. Mir schien letztere aber philosophisch zu voraussetzungsreich, um sie auch Nicht-Philosophen in einem Aufsatz verständlich zu machen. In meiner Skizze werde ich zuerst Pädagogik als Wissenschaft konstituieren und dann notwendigerweise zur Konstitution der Subdisziplin Medienpädagogik übergehen.

\section{Grundlegung von Pädagogik als Wissenschaft und deren Probleme}

Mein erstes Axiom oder schlichter: mein erster Grundsatz ist, Bildung ist Gegenstand der Pädagogik.

Wenn der Bildungsbegriff für die Pädagogik als Wissenschaft und als Praxis leitend ist, dann hat Erziehungswissenschaft das Problem zu behandeln, dass der Einzelne ein Selbst- und Welt-Verhältnis ausbildet. Dass Bildung irgendetwas mit dem Selbstund Weltverhältnis zu tun hat, ist ein pädagogischer Gemeinplatz (topos). Wie dieses

4 Ob es Dämonen gibt oder nicht (Dass-Sein), wird damit nicht ausgesagt. Die Begriffsentwicklung konstituiert das Wesen, das Was-Sein, unsterblich und unvernünftig. Wenn unsterblich und unvernünftig etwas definiert, dann kann man für dieses etwas den Ausdruck Dämon einführen. 
Verhältnis zu gestalten ist, mag kontrovers sein. Aber dass es bei Bildung um dieses Verhältnis geht, ist in der Pädagogik unbestritten.

Also hat die Erziehungswissenschaft dieses Sich-Verhalten zur Welt und zu sich selbst zu analysieren, empirisch zu erforschen und auf seine Folgen zu untersuchen. In dieses Verhältnis, das der Einzelne ausbildet, gehen mindestens 4 Begründungsfelder notwendig ein. Das ergibt sich aus meiner Ausdifferenzierung des Selbst- und Weltverhältnisses in das dreifache Verhältnis der Bildung (Meder 2007). Ich formuliere hier die als minimal zu bezeichnenden Bedingungen, ohne die oder ohne eine Positionierung auf sie keine Erziehungswissenschaft grundgelegt werden kann.

a. Der Einzelne ist ein Mensch, also wird es anthropologische Voraussetzungen geben. Denn Anthropologie ist die Theorie vom Menschen in seinen Gattungsmerkmalen.

b. Da der Einzelne zum ersten ein Verhältnis zu den Sachen und Sachverhalten in der Welt ausbilden muss, gehen erkenntnistheoretische Voraussetzungen ein. Denn das Verhältnis zu den Sachen und Sachverhalten steht unter dem Kriterium der Wahrheit. Was Wahrheit ist oder sein kann, wird in der Erkenntnistheorie (bzw. auch in der Wissenschaftstheorie) behandelt.

c. Da der Einzelne zum zweiten ein Verhältnis zu den anderen in der Gemeinschaft ausbilden muss, muss vorausgesetzt werden, was die Gemeinschaft ist. Deute ich die Gemeinschaft als Kulturgemeinschaft, dann bedarf es einer kulturwissenschaftlichen bzw. einer kulturphilosophischen Begründung. Deute ich die Gemeinschaft als Gesellschaft, dann bedarf es einer soziologischen, d. h. heute üblicherweise einer sozial-strukturellen Begründung. Deute ich die Gemeinschaft als Staat, dann bedarf es einer staatstheoretischen, politischen Begründung.

d. Da der Einzelne zum dritten ein Verhältnis zu sich selbst auszubilden hat, dies aber nur in den Strukturen der Zeit möglich ist, d. h. als Bezug auf seine Vergangenheit (Biografie) oder als Bezug auf seine Zukunft (Selbstentwurf, Hoffnung und Ideale) oder auch aktual als Bezug und Stellungnahme auf seine gegenwärtige Situation, bedarf es einer zeittheoretischen Begründung. Sie kann als psychologische Theorie des Zeitbewusstseins geliefert werden. Sie kann aber auch als Theorie der Zeit und Geschichte überhaupt zur Darstellung kommen. In jedem Falle kommt hier das normative, moralische Problem zur Sprache. Es entsteht die Frage nach dem Sinn des Lebens. Wie kann und darf der Einzelne seine Zukunft entwerfen? Welchen Sinn darf er sich geben und welchen nicht? Auf welche Selbstverwirklichung darf er aus sein und welche Selbstverwirklichung - etwa als Berufskiller - ist unzulässig. Dabei spielen gleichwohl die Prämissen der anderen Grundlegungen eine massgebliche Rolle. 


\section{Anthropologische Grundlegung}

Anthropologische Grundlegungen der Erziehungswissenschaft stehen grundsätzlich unter Ideologieverdacht. Man denke nur an die neuere Gender-Debatte. Denn über angebliche Gattungsmerkmale könnte prädisponiert werden, was mit dem einzelnen Menschen zu machen ist und was der Einzelne mit sich selbst machen kann und muss. Erziehungswissenschaftlich können aber diese Fragen nicht in der Grundlegung vorentschieden werden. Denn sie sollen ja empirisch erforscht werden. Ihre Antworten sollen Ergebnis erziehungswissenschaftlicher Forschung sein. Also kann und darf man sie nicht schon in die Grundlegung stecken. Wir brauchen als Erziehungswissenschaftler nur voraussetzen, dass der Mensch instinktreduziert, also instinktarm ist. Daraus resultiert, dass er offen ist, seine Umwelt als Welt zu definieren (vgl. Plessner 1976) ${ }^{5}$. In solchen Definitionen ist der Mensch an vorangegangene Definitionen verwiesen. Dies macht seine Geschichtlichkeit und seine Kulturalität aus. Mit Bezug auf seine geschichtliche Kultur ist auch die Möglichkeit gegeben, ihn «von aussen` zu bilden (Bildsamkeit). Der Einzelne kann die Überlieferungen übernehmen oder sich negativ zu seiner Geschichtlichkeit verhalten. Er kann opponieren und alles ganz anders machen, d. h. er kann sich radikal selbst bilden (Bildsamkeit). Was er allerdings nicht kann, ist sich zur Überlieferung nicht zu verhalten - sieht man mal von den sogenannten Wolfskindern ab.

Man muss also aus der Anthropologie des 20. Jahrhunderts - unabhängig von den jeweiligen Detailforschungen - übernehmen, dass der Mensch naturhaft dazu bestimmt ist, sich selbst in Auseinandersetzung mit der jeweiligen Kultur in einer ökologischen Nische zu bestimmen. Der Zwang zur Selbstbestimmung ergibt sich aus der mangelnden Instinktsteuerung. Eine darauf aufsetzende Pädagogische Anthropologie versteht diesen Mangel positiv als Chance - eben als Bildsamkeit. Es ist wichtig zu sehen, dass bei diesen Voraussetzungen noch keinerlei dogmatische oder normative Vorentscheidungen getroffen sind.

\section{Erkenntnistheoretische Grundlegung}

Die erkenntnistheoretische Grundlegung wird in wesentlichen Teilen auch in die kulturwissenschaftliche Grundlegung eingehen. Sie geht auf Kant und insbesondere auf den Neukantianer Hönigswald zurück (Hönigswald 1927). In ihrer Reinform, d. h. ohne Bezug auf Kultur, lautet sie folgendermassen: Da der Mensch nicht instinktgesteuert an eine bestimmte Umwelt gebunden ist, muss er sich die Umwelt schaffen. Das kann er nur, wenn er sich mit den gegebenen Sachen und Sachverhalten auseinandersetzt und sich sach-adäquat verhält. Dazu bedarf es einer Orientierung bzw. eines Entscheidungskriteriums dafür, was gültig ist. Dies ist Wahrheit. Das Streben nach, das Aus-Sein auf Wahrheit kann nie wissen, ob Wahrheit endgültig erreicht ist.

5 Recht gelesen ist Plessners Anthropologie die beste Pädagogische Anthropologie, die ich kenne. 
Es ist stets denkbar, dass Gegenbeispiele, Gegenkonzepte, Widerlegungen möglich sind. Der Erkennende ist sich seiner Erkenntnis niemals ganz sicher, weil er als endlicher die Form seiner Begrenztheit nicht kennen und nicht wissen kann. Er müsste ja wissen, was er nicht weiss. Das ist prinzipiell nicht möglich, weil es eine Paradoxie ist. Stattdessen kann er nur wissen, dass er vieles nicht weiss. Das Viele, was er nicht weiss, ist ihm nur als unbestimmte Limitation bewusst. Also muss das, was er für wahr hält, einer unendlichen Geltungsbewährung ausgesetzt werden.

Der pädagogische Handlungszusammenhang ist nun der soziale Ort, an dem diese unabschliessbare Geltungsbewährung faktisch stattfindet. Man könnte einwenden, dass das doch das Geschäft der Wissenschaft ist. Der Einwand ist richtig. Aber indem Wissenschaft ihre Ergebnisse - das sind bis auf weiteres wahre Sätze - zur Disposition stellt, begibt sie sich in den Modus des pädagogischen Handlungszusammenhanges. Nichts anderes meint ja das Motiv der Einheit von Forschung und Lehre. Was als wahr in der Forschung entwickelt wird, fordert als solches auch die faktische Anerkennung aller. Was sollte sonst Wahrheit heissen, wenn auf ihre Anerkennung seitens aller verzichtet werden könnte? Also fordert der Anspruch auf Wahrheit seine Bewährung im pädagogischen Handlungszusammenhang.

Wahrheit meint im Geltungsanspruch natürlich primär, dass der wahre Satz die wirklichen Sachverhalte abbildet (adaequatio intellectus et $\mathrm{re}^{6}{ }^{6}$ ). Man nennt dies auch die Validität eines wissenschaftlichen Satzes. Man prüft sie, indem man die Perspektiven und Methoden, die Sachverhalte zu betrachten und zu erarbeiten, wechselt. Erhält man trotz Wechsel der Perspektive und der Methoden dieselben Ergebnisse, d. h. denselben 'wahren` Satz, dann gilt dies als Validitätsprüfung. Bei genauerem Hinsehen handelt es sich dabei aber nur um eine Annäherung. Denn um zu prüfen, ob ein Satz die wirklichen Sachverhalte abbildet, müsste man in der Lage sein, beide Seiten der Abbildung - das Original und das Abbild, die Wirklichkeit und den Satz - in den Blick zu nehmen. Aber genau das können wir in unserer endlichen Erkenntnis nicht. Ein solches Vermögen wird in der westlichen Kultur nur Gott zugeschrieben. Die genannten Validitätsprüfungen sind mithin nur Behelfe, mit dem Problem angesichts unserer Endlichkeit umzugehen.

Damit kommt ein weiteres Kriterium für Wahrheit ins Spiel. Die Validitätsprüfung eines «wahren` Satzes lässt sich nicht abschliessen, weil es einen unüberschaubaren Vorrat von Perspektiven und Methoden gibt. Diese können nur in Annäherung eingebracht werden, wenn alle sich an der Geltungsbewährung beteiligen. Denn es sind die unterschiedlichen Subjekte, die die unterschiedlichen Perspektiven und Methoden einbringen. Dieser Umstand führt zum Kriterium der Intersubjektivität von Wahrheit: Ein Satz ist nur dann wahr, wenn er bei gleicher Perspektive und bei gleichen Methoden von allen Subjekten der Forschung bestätigt wird. Und zugleich: ein Satz ist nur dann valide, wenn er im Perspektiven- und Methodenwechsel aller sozialen

6 Sinngemäss: Adäquation (Entsprechung) von Erkenntnis und Sache. 
Partner als gleiches Ergebnis resultiert. Mit allen sozialen Partnern sind nicht nur die gegenwärtig Lebenden gemeint, sondern auch alle zukünftigen. Damit ist der dritte Aspekt von Wahrheit angesprochen: Ein wahrer Satz muss wahr sein zu aller Zeit. Man nennt dies die Reliabilität der Wahrheit. Ein wahrer Satz muss auch wahr sein für die nachvollziehenden Perspektiven und Methoden zukünftiger Menschen. Er muss auch wahr sein bei wechselnden Perspektiven und Methoden zukünftiger Generationen von Forschern und Alltagsmenschen. Spätestens an dieser Stelle geht der Wahrheitsauftrag bzw. allgemeiner der Geltungsanspruch in den pädagogischen Handlungszusammenhang ein.

Man sieht an diesen Überlegungen, dass sich das Problem der Geltungsbewährung in drei Dimensionen gliedert: die Sachdimension (Validität), die Sozialdimension (Intersubjektivität/Objektivität) und in die Zeitdimension (Reliabilität). Wir haben schon gesehen, dass diese Gliederung auch - in einem noch weiteren Sinne - in den Bildungsbegriff eingeht. Wenn es im pädagogischen Handlungszusammenhang wirklich um faktische Geltungsbewährung geht, dann kann das auch nicht anders sein.

Für eine geltungstheoretische Grundlegung der Pädagogik als Wissenschaft kann festgehalten werden: Pädagogisches Handeln ist der Ort, an dem die faktische Geltung von Geltungsansprüchen der alten Generation in Auseinandersetzung mit der Neuen Generation ausgehandelt wird. Die Situation der Aushandlung ist grundsätzlich geltungsoffen.

\section{Kulturwissenschaftliche Grundlegung der Pädagogik?}

Wir alle werden in eine schon kulturell (vor-)bestimmte Welt geboren, die wir uns nicht auswählen können. Erziehung kann als Einführung in die historisch gegebene Kultur verstanden werden. Bildungsprozesse sind in der Korrelation, im Zusammenhang, von «Sich-bilden` und ‘Alles-andere-bildet-mich〉 zu begreifen. Bildungsprozesse sind Auseinandersetzungen mit den kulturellen Vorgaben, über die wir einerseits nicht verfügen, aber über die wir andererseits Kontroversen und Konflikte im pädagogischen Handlungszusammenhang austragen. Man wählt im kulturellen Horizont unbestimmt vieler Alternativen das, was man für das Richtige hält. Das, was man für richtig hält, soll gelten, soll gültig sein. Es wird in einem Geltungsanspruch formuliert und muss sich bewähren. Im kulturellen Spiel um Geltungsbewährung geht es stets um das, was auf Dauer Bestand haben soll. Wenn wir Überlieferung als die Akte der Geltungsbewährung verstehen, dann ist das Generationenproblem der Ort solcher Geltungsbewährung. In modernen Gesellschaften ist das Bildungs- und Erziehungssystem entwickelt worden, um genau dieses Problem zu bearbeiten. Hönigswald hat es so gesagt: Es geht in der Pädagogik um die Überlieferung der Geltungsansprüche an die übernächste Generation vermittels der nächsten. Damit hat er am präzisesten das Generationenproblem charakterisiert. Die nächste Generation muss zu Erziehern 
der übernächsten Generation erzogen werden. Nur so kann rekursiv die Dauer des Geltungsanspruchs gesichert werden.

So und nur so kann Kultur etwa gegen Zivilisation bestimmt werden. Denn Zivilisation ist durch den Status des Bürger-Seins (Civis) bestimmt. Dieser Status kann verfassungsrechtlich (USA, Frankreich), ethnisch (Deutschland), religiös (Israel) oder sonst wie bestimmt sein. Dagegen ist die kulturelle Bestimmtheit einer Gemeinschaft relativ unabhängig von der Gesellschaftsform und der Staatsform. Die jeweilige Kultur bestimmt sich über die Antworten auf die Frage nach dem richtigen Leben in der gegebenen Umwelt. Die jeweilige Weltsicht, die je geltenden Normen und Werte und deren symbolische Darstellung von Wahrheit machen kulturelle Bestimmtheit aus. Kulturelle Bestimmtheit der Gemeinschaft ist mithin stets Geltungsbestimmtheit der Gemeinschaft.

\section{Hannah Arendts kulturwissenschaftliche Deutung der Pädagogik}

Weil dies so ist, ist der pädagogische Streit zwischen Neuen (Neugeborenen) und Alten (Alteingesessenen) rein formal gesetzt. Dies hat Hannah Arend in ihrem Vortrag «Über Erziehung» von 1957 herausgestellt. Wenn die Geltungsfrage prinzipiell offen ist, wie das in dynamischen Gesellschaften der Fall ist, dann gestaltet sich Erziehung und Bildung im Modus der Auseinandersetzung. Der Generationenwechsel gestaltet sich also in der kommunikativen Auseinandersetzung um Kultur, d. h. in der bipolaren Kontroverse von Bewahrung und Innovation. Denkt man dies im Paradigma des Generationenwechsels (d.h. im Zeit-Aspekt) weiter, dann geht es im Namen der Geltung um die Mediatisierung der nächsten Generation. Sie muss nicht nur den Geltungsanspruch des kulturellen Lebens bewähren, sondern auch als Geltungsanspruch im Diskurs halten. Sie muss das Medium der Prozessualisierung von Geltungsansprüchen sein, d. h. der Träger zeitlicher Vollzüge der Geltung. In der Nachfolge von Chomsky wird die Vollzugsqualität eines Regelwerks auch Performanz genannt. In diesem Sinne kann die je nächste Generation auch als der performante Operator von Geltungsansprüchen genannt werden. Die nächste Generation muss stets als die nächste Generation von Erziehern und pädagogisch Handelnden betrachtet werden, damit die übernächste Generation immer noch mit denselben Geltungsansprüchen des Kulturellen konfrontiert ist.

Darin hat Hönigswald Recht und geht über alle Generationentheoretiker hinaus, wenn er sagt, dass Überlieferung von Kultur und Geltungsansprüchen in der Kultur nur an die übernächste Generation vermittels der nächsten Generation möglich ist. Und das gilt prinzipiell, weil sich sonst keine Rekursion und damit keine Kontinuität entwickeln kann. Und es gilt auch faktisch, weil meine Kinder und meine Schüler und meine Seminarteilnehmer in der Weiterbildung das weitertragen sollen, was ich für relevante Geltungsansprüche halte. 
Denkt man dieses Paradigma des Generationenwechsels im sozialpädagogischen Paradigma der Innen-Aussen-Differenz weiter, dann geht es im pädagogischen Handlungszusammenhang darum, die Mediatisierung dessen, der draussen steht, derart zu befördern, dass er die ‘Schnittstelles für weitere Integration wird. Konkret gesprochen: der Streetworker versucht den Randständigen (Obdachlosen, Junkie etc.) in die Gemeinschaft zurückzuholen und ihn gleichzeitig mental darauf vorzubereiten, dass er die Erfahrung seiner Rückkehr in die Gemeinschaft einem anderen Randständigen so vermitteln kann, dass auch er in die Gemeinschaft zurückkehrt. In dieser Mediatisierung - sei es im Generationenwechsel oder bei dem Problem von Inklusion und Exklusion - wird der gesellschaftliche Bildungsprozess auf Dauer gestellt. Damit ist die Strategie pädagogischen Handelns strukturell geklärt, offen bleibt, wie diese Strategie auf der Interaktionsebene umzusetzen ist.

Für eine Grundlegung der Erziehungswissenschaft bleibt festzuhalten, dass auch die kulturwissenschaftlichen Grundannahmen für die Offenheit des Bildungsprozesses vor dem Hintergrund der Kultur sprechen.

\section{Was ist die soziologische Grundlegung?}

Für die moderne Pädagogik als Praxis und Wissenschaft bzw. für die Erziehungswissenschaft ist entscheidend, dass sich die abendländische Gesellschaft im 18. Jahrhundert in verschiedenen historischen Strängen von einer ständischen, zunftorientierten und feudalen Gesellschaft (von einer so genannten statischen bzw. stratifikatorischen Gesellschaft) hin zu einer funktional differenzierten Gesellschaft entwickelt hat.

Nach Ständen organisierte Gesellschaften gelten als statisch, weil gesellschaftliche Aufgaben - wie das Backen von Brot, das Brauen von Bier, das Regieren - bestimmten fest umrissenen Gemeinschaften überlassen bleiben. Als Sohn eines Bäckers wirst du wieder Bäcker, als Sohn eines Bierbrauers wirst du wieder Bierbrauer, als Sohn eines Königs wirst du wieder König.

Dynamisch sind die westlichen Gesellschaften erst dadurch geworden, dass die bürgerlichen Stände revolutionär durchgesetzt haben, dass derjenige eine gesellschaftliche Funktion verrichtet, der es am besten kann. Der wird König, der am besten regieren kann. Der wird Bäcker, der am besten Brötchen backt, der wird Bierbrauer, der das beste Kölsch braut. Für diesen Denkansatz ist insbesondere Rousseau in seinen sozial- und politisch-kritischen Schriften, aber auch im «Emile» progressiv gewesen. Und viele Historiker vermuten hinter ihm den intellektuellen Wegbereiter der französischen Revolution (vgl. Meder 2014).

Funktional differenzierte Gesellschaften kultivieren die Norm, dass der Bestmögliche eine vakante Funktionsstelle in der Gesellschaft einzunehmen hat. Um den Bestmöglichen zu finden, muss gesellschaftlich Konkurrenz etabliert und kultiviert 
werden. Darüber hinaus muss der Einzelne internalisieren, dass es auf ihn ankommt in der Gesellschaft, aber nur insoweit als er sein Optimum entwickelt. Selbstverwirklichung wird zum Diktat, aber auch zur Gefahr, sofern sie nicht in der Richtung verläuft, in der sie gesellschaftlich gefordert ist.

Aus dieser soziologischen Begründung muss die Pädagogik als Wissenschaft übernehmen, dass Bildung bipolar konzipiert ist. Bildung steht in der Bipolarität von gesellschaftlichen Bedürfnissen bzw. Zwängen einerseits und der individuell geprägten Selbstverwirklichung einzelner Menschen andererseits. Diese Bipolarität ist äusserst konträr und konfliktgeladen. Sie ist aber auch der Motor des Fortschritts funktional differenzierter Gesellschaften. Insofern muss sie gesellschaftlich ausgehalten werden. Dass dies geschieht, dafür ist das Bildungs- und Erziehungssystem zuständig. Seine Aufgabe ist es die individuellen Entwicklungstendenzen mit den Tendenzen gesellschaftlicher Entwicklung strukturell zu koppeln. Da es für eine solche strukturelle Kopplung keine Regel gibt, haben professionelle Pädagogen ein hohes Mass an Unsicherheit und Risiko zu bearbeiten und auszuhalten.

Diese Unsicherheit und dieses Risiko, die in Folge der aufgezeigten gesellschaftlichen Veränderungen entstehen, werden in der historischen Entwicklung zum Problem des Bildungs- und Erziehungssystems. Durkheim, Simmel, Parsons, Mead und dann auch Luhmann haben genau dieses Problem vor Augen, wenn es um soziologische Aspekte der Erziehung und Bildung geht.

Die Grundlegung der Pädagogik als Wissenschaft kann hier den Gedanken übernehmen, dass die Aushandlung von gesellschaftlichen und individuellen Ansprüchen an ein gutes Leben grundsätzlich offen ist. Für eine Grundlegung der Erziehungswissenschaft braucht also nur festgehalten zu werden: Die Abgleichung individueller Selbstverwirklichung mit den gesellschaftlichen Anforderungen ist prinzipiell ein offener Prozess (vgl. Simmel 1922, Meder 2007a).

\section{Was ist eine zeittheoretische Grundlegung?}

Schon der Generationenkonflikt ist unter dem Gesichtspunkt von Zeit zu analysieren. Des Weiteren ist die Reliabilität der Wahrheit ein zeittheoretisches Wahrheitskriterium. Dies sind Aspekte der Grundlegung, die es nahelegen, über Zeitprämissen für eine Theorie der Pädagogik nachzudenken. Dennoch haben wir oben das Zeitproblem vor allem im Selbstverhältnis verankert. Im Verhältnis zu mir selbst - vergangenheitsbezogen oder zukunftsbezogen - gibt es zwei Zeitpunkte, die meiner Lebensgestaltung prinzipiell entzogen sind. Das sind meine Geburt und mein Tod. Kein anderes Lebewesen weiss um seinen Tod. Das Wissen um den eigenen Tod kann deshalb als ein anthropologisches Merkmal angenommen werden. Um den eigenen Tod zu wissen, wirft die Frage nach dem Sinn des Lebens auf. Unter Zeitgesichtspunkten bedeutet die Sinnfrage, wie ich die Lebenszeit inhaltlich ausfüllen soll: Was heisst für 
den je Einzelnen erfüllte Zeit? Was heisst leere Zeit, Leerlauf oder gar Verzweiflung über die nicht sinnvoll genutzte Zeit. Wenn man die Zeit zwischen Geburt und Tod, den unverfügbaren Grenzen, als den Lebenslauf bezeichnet, dann gilt es diesen Lebenslauf als je eigene Biografie zu formen. Dabei ist der Lebenslauf nicht einfach eine leere Zeitform, die es zu füllen gilt. Lebenslauf ist vielmehr immer schon durch biologische und kulturspezifische Regelmässigkeiten vorstrukturiert. In diesen Strukturen sind inhaltliche Möglichkeiten zeitbedingt prädisponiert. So lernt man Sprache und Sprachen am besten zwischen dem 2. und 6. Lebensjahr, und die Midlife-Krise ereignet sich am ehesten zwischen dem 50. und 60. Lebensalter, wenn man meint, das erreicht zu haben, was möglich war und prüft, ob es den früheren Lebenshoffnungen entspricht.

Lebenslauf ist also keine leere Form, sondern eine vorstrukturierte Form, wobei die Struktur durch mögliche Entscheidungsräume in bestimmten Lebensphasen bestimmt ist. Die Aufgabe für den Einzelnen ist, in diesen Möglichkeitsräumen für sich selbst die passenden Entscheidungen zu treffen und damit dem Lebenslauf seine individuelle Gestalt zu geben. In Anlehnung an Hönigswald kann eine solche Gestaltgebung als die Abbildung dessen, was man für richtig erkannt hat, in die Zeit verstanden werden. Was man für richtig erkennt, ist für sich genommen nicht zeitlich. Man erkennt es als richtig aus sachlogischen Gründen, die zu aller Zeit zu gelten beanspruchen (Reliabilität der Wahrheit). Die sachlogischen Zusammenhänge müssen nun in die eigene Lebenszeit abgebildet werden, damit sie sich aktual zeigen, in meinem Leben performant werden und als Geltungsanspruch für alle lebendig sind. Das bedeutet für Hönigswald, dass sich das für wahr Beanspruchte nicht nur in die aktuelle Lebenszeit eines Einzelnen abbilden muss, sondern auf die ganze Zeit der Geltungsbewährung in einer Kulturgemeinschaft.

Was man aus den zeittheoretischen Grundgedanken für eine Grundlegung der Pädagogik als Wissenschaft festhalten muss, ist der Abbildungsgedanke. Ein sachlogisch Strukturiertes, das als richtig angenommen wird, muss in den zeitlichen Verlauf des Lebens eines Einzelnen abgebildet werden. Diese Abbildung ist nicht eindeutig bestimmbar, da sachlogische Ordnungen netzartig sind, die zeitliche Ordnung aber linear. Die Abbildung muss zwar geleistet werden, ist aber in der Art und Weise ihrer Realisierung grundsätzlich offen. Herbart charakterisierte das Problem dieser Abbildung als die ästhetische Darstellung der Welt als das Hauptgeschäft der Erziehung. Die Abbildung als ästhetisch zu bezeichnen, heisst, dass sie offen für jede performante Verwirklichung in irgendeiner Zeit ist. 


\section{Das Gemeinsame aller Grundlegungen}

Alle Grundlegungen kommen darin überein, dass aus je unterschiedlichen Perspektiven die Ausgangssituation für die menschliche Entwicklung nicht determiniert und damit offen ist. Diese Offenheit bezeichne ich in der Tradition pädagogischer Theorie als Bildsamkeit. Bildsamkeit heisst im Einzelnen entlang der Grundlegungsvarianten anthropologische Unterbestimmtheit des Instinktiven, soziologisch keine Vorbestimmung des gesellschaftlichen Platzes eines Individuums ${ }^{7}$, kulturwissenschaftlich Unsicherheit des Ausgangs der Überlieferung, geltungstheoretisch offene Auseinandersetzung um Geltungsbewährung und zeittheoretisch ästhetische und damit nicht-deterministische Gestaltung des Lebenslaufes. Alle diese Grundannahmen und Grundgedanken sind nicht metaphysisch, sondern empirisch belegt. Sie konstatieren Unterbestimmtheit, Unbestimmtheit und Offenheit, aber keine normative Richtung, mit diesem Umstand umzugehen. Damit sind sie geeignet, als Ausgangspunkt einer allgemeinen und systematischen Pädagogik als Wissenschaft fungieren zu können. Zusammenfassend seien diese Grundannahmen Bildsamkeit genannt. Bildsamkeit hat mithin einen anthropologischen, einen soziologischen, einen kulturwissenschaftlichen, einen geltungstheoretischen und einen zeittheoretischen Aspekt. Diese explizit pluralsperspektivische Sicht unterscheidet meinen Begriff von Bildsamkeit von dem der pädagogischen Tradition.

\section{Grundlegung der Medienpädagogik}

Die Struktur des Bildungsbegriffs war der Leitfaden der hier vorgestellten Skizze einer Grundlegung. Diese Struktur stellt ein Beziehungsgefüge dar, das ich das dreifache Verhältnis der Bildung genannt habe.

a. Einzelner / Sachen und Sachverhalten / in der / Welt

b. Einzelner / Anderer und Andere / in der / Gemeinschaft

c. Einzelner / er selbst / in der / Lebenszeit

Dieses Beziehungs- oder Relationsgefüge hat Netzstruktur. Statt von einem Bildungsverhältnis müsste man genauer von einem Bildungsnetz sprechen, in dem der Einzelne als zentraler Knoten unter drei differenzierbaren Aspekten mit seiner Umwelt in Beziehung gesetzt ist. Dabei war stillschweigend vorausgesetzt, dass es sich dabei um ein dynamisches Beziehungsverhältnis handelt. Das, was struktural logisch als das Relationale im Bildungsnetz gefasst wird, steht für empirische Ereignisse, d. h. für Auseinandersetzungen in kognitiven Aktivitäten, für Auseinandersetzungen in sozialen Handlungszusammenhängen und für Auseinandersetzungen im Ringen um

7 Dass etwa die soziale Schicht die Bildungschancen und damit die Bildsamkeit beeinflusst, ist ein empirisches Phänomen und wird gerade im Rahmen solcher Grundlegung theoretisch fassbar. 
ein sinnvolles Leben. Man kann abgekürzt sagen, dass das Relationsgefüge für eine soziale Praxis steht.

Diese soziale Praxis ist - wie ich gezeigt habe - Auseinandersetzung, Konflikt und Streit in drei Dimensionen bzw. unter drei Aspekten: erstens Streit um Geltung, zweitens Generationenkonflikt in Sachen Kultur, Politik und Abgleich von Individuum und Gesellschaft sowie drittens Selbstverwirklichung im Modus der Sinnfrage.

\section{Das konstitutive Moment der Darstellungsmedien}

Die Bedingung der Möglichkeit der Auseinandersetzung oder des Streites um Geltung ist die Differenz von Geltungsanspruch und Wirklichkeit, die in der adaequatio intellectus et rei aufzuheben ist. ${ }^{8}$ Diese Differenz kann keine strukturelle und auch keine sachlogische sein, denn dann könnte es gar keine Adäquation geben. Die Differenz muss daher die Materie betreffen. Die Materie der Wirklichkeit ist eine andere als die des Geltungsanspruches. Nur deshalb kann man sagen, dass der Geltungsanspruch die Wirklichkeit darstellt. Darstellung heisst, dass die Materie in der Darstellung stellvertretend fungiert für die Materie des Dargestellten. Durch diese Repräsentationsfunktion wird die Materie der Darstellung zum Zeichen-für und damit zum Medium. Die erkenntnistheoretische bzw. die geltungstheoretische Konstitution der Pädagogik lebt von dem konstitutiven Moment des Darstellungsmediums. Was nicht dargestellt werden kann, kann auch nicht erkannt und in einem Geltungsanspruch formuliert werden. Das heisst: Mit der erkenntnistheoretischen Grundlegung der Allgemeinen Pädagogik ist zugleich die Grundlegung der Medienpädagogik mit Bezug auf Darstellungsmedien mit vollzogen. Konkret mündet das in den Komplex der didaktischen Medien, denn sie sind die Darstellungsmedien, in denen der Geltungskonflikt praktisch ausgetragen wird.

\section{Das konstitutive Moment der Kommunikationsmedien}

Die Bedingung der Möglichkeit des Generationenkonfliktes ist die Differenz, dass die Neuen die Alteingesessenen nicht verstehen und umgekehrt. Zur Überwindung dieser Differenz bedarf es eines Mediums der Verständigung. Das ist ein Medium, in dem das wechselseitige Verstehen ausgehandelt werden kann, das dabei allerdings niemals garantiert, dass man sich wirklich versteht. Es ist wie mit dem Darstellungsmedium: Ob es die Wirklichkeit adäquat abbildet, ist nie abschliessbar zu entscheiden. Ob ein Medium der Verständigung das, was ein Individuum meint, adäquat zum Ausdruck bringt, ist ebenso nicht entscheidbar.

Unter dem erkenntnis- und geltungstheoretischen Aspekt mit all seinen didaktischen Implikationen ist man traditionell stets auf Sprache als das basale Medium der

8 Hier wird meine Grundlegungsskizze rekursiv im Sinne einer transzendentalen Argumentation. 
Darstellung verwiesen worden. Dabei wird häufig übersehen, dass der Leib im Medium seiner Sensomotorik ein entscheidendes Moment der Geltungsbewährung ist. Bei Verständigungs- und Kommunikationsmedien spielt der Leib eine noch gewichtigere Rolle. Im frühkindlichen und damit vorsprachlichen Verständigungsprozess zwischen den Generationen ist der Leib in seinem sensomotorischen Habitus das alleinige Medium der Verständigung. Deshalb findet in dieser Auseinandersetzung der Generationen Inkorporation oder autistische Abwehr statt. Deshalb geht es in dieser Auseinandersetzung vor allem auch um die leiblich dispositionelle Einstellung zu Welt und Gemeinschaft, die Erickson im Konflikt zwischen Urvertrauen und Misstrauen beschrieben hat (vgl. Meder 2013). Diese Andeutung soll deutlich machen, dass im Medium der Verständigung ein sehr viel breiteres Spektrum an Medien relevant ist als nur die Sprache.

\section{Das konstitutive Moment der Interaktionsmedien}

Die Bedingung der Möglichkeit der Selbstfindung und Selbstverwirklichung ist der Umstand, dass man nicht wissen kann, wer man ist. Differenztheoretisch ausgedrückt ist die Bedingung der Möglichkeit der Selbstverwirklichung die Differenz von Wissen-wollen und Nicht-Wissen. Das sogenannte Selbst ist nicht nur den anderen unbekannt, sondern auch dem Einzelnen selbst. Das liegt einerseits daran, dass man über seine Geburt und die damit verbundene Prägung nichts unmittelbar weiss, und andererseits noch viel ausschlaggebender, dass man über seine Zukunft und seinen Tod nur begrenzt verfügen kann. Wer und was man also selbst ist, muss man mit sich selbst aushandeln. Das dazu notwendige Medium ist das Interaktionsmedium. In ihm entwerfe ich mich, mache eine Darstellung von mir mit der besonderen Perspektive, im Modus dieser Selbstdarstellung mit meiner Umwelt zu interagieren. Ich versuche mich, ich teste mich, ich erfinde mich im Modus von Versuch und Irrtum. Genau dies ist der Grund, dieses Medium gesondert zu bezeichnen. Natürlich können alle diese Selbstversuche in den Medien der Verständigung und im geltungstheoretischen Medium der Darstellung stattfinden. Aber indem die Auseinandersetzung mit mir selbst nur erfolgreich ist, wenn ich ein Selbst-Test-Ergebnis erhalte, ist hier ein anderer Aspekt dominant.

Conclusio: Medialität als notwendiges Konstituens Allgemeiner Pädagogik und als Alleinstellungsmerkmal der Medienpädagogik

Indem man die Relationalität im Bildungsverhältnis thematisiert, ist man unweigerlich auf die Medialität aller Prozesse der Bildung verwiesen. Das macht eigentlich die Medienproblematik zu einem Teil der Allgemeinen Pädagogik. Das heisst, dass das Problem der Medialität jede Bildungssituation und jeden pädagogischen 
Handlungszusammenhang betrifft. Sofern sich Medienpädagogik genau dieser Problematik der Medialität alles Pädagogischen annimmt, ist sie notwendig eine Querschnittsdisziplin, die in allen Subdisziplinen der Pädagogik - gleichgültig was immer das «Sub» bedeutet - vorkommen muss. Deshalb muss sich auch jede Subdisziplin - ob es die Schulpädagogik, die Didaktik, die Sozialpädagogik, die Behindertenpädagogik oder die Erwachsenenbildung ist - sich mit den Medien der Darstellung, der Verständigung und der Interaktion in ihrem Bereich beschäftigen. Weil aber solche Beschäftigung in den Subdisziplinen immer auf das spezifische eigene Problem hin beschränkt ist, bedarf es einer Teildisziplin, die sich dem Problem der Medien in allen Bildungssituationen und pädagogischen Handlungszusammenhängen annimmt. Gegenstand einer solchen Teildisziplin ist natürlich jedes Medium, das in pädagogischen Handlungszusammenhängen vorkommen kann. Diese Gegenständlichkeit teilt die Medienpädagogik aber mit je verschiedenen Subdisziplinen, kann darin jeweils wissenschaftlich kooperieren und jeweils alternative Möglichkeiten aus anderen Subdisziplinen ins Spiel bringen.

Was aber der eigentliche Gegenstand der Medienpädagogik ist, ist die Medialität des Pädagogischen. Genau besehen ist das eigentlich ein Thema der Allgemeinen Pädagogik. Die Allgemeine Pädagogik als Subdisziplin in ihrer gegenwärtigen Ausprägung und in ihrer gegenwärtigen empirischen Präsenz bzw. Erscheinungsform ist allerdings nicht in der Lage dieses Problem zu behandeln. Von daher ist es notwendig, dass sich eine gesonderte Teildisziplin um das allgemeinpädagogische Problem der Medialität in Bildungssituationen und in pädagogischen Handlungszusammenhängen zum Gegenstand macht und in speziellen Forschungen ihre disziplinäre Identität findet. 


\section{Literatur}

Hogrebe, Wolfram. 1971. «Konstitution». In Historisches Wörterbuch der Philosophie, herausgegeben von Joachim Ritter, Karlfried Gründer, und Gottfried Gabriel, 4. Band (I-K):9921004. Basel: Schwabe Verlag. http://dx.doi.org/10.24894/HWPh.5212.

Hönigswald, Richard. 1927. Über die Grundlagen der Pädagogik. München: Reinhardt.

Meder, Norbert. 2007. «Der Lernprozess als performante Korrelation von Einzelnem und kultureller Welt. Eine bildungstheoretische Explikation des Begriffs». Spektrum Freizeit 1-2: 119-135. http://nbn-resolving.org/urn:nbn:de:hbz:464-20160706-091750-5.

Meder, Norbert. 2007a. «Die Grenzen der Erziehungswissenschaft sind Absolut». Vierteljahrsschrift für wissenschaftliche Pädagogik 83 (4): 434-447.

Meder, Norbert. 2013. Habitus - auch medialer Habitus - aus pädagogischer Perspektive. In MEDIENIMPULSE 04/2013. http://www.medienimpulse.at/articles/view/599.

Meder, Norbert. 2014. «Das Bildungskonzept als politischer Kampfbegriff». In Kritik-Bildung - Forschung. Pädagogische Orientierungen in widersprüchlichen Verhältnissen, herausgegeben von Harald Bierbaum, Carsten Bünger, Yvonne Kehren, und Ulla Klingovsky, 217-36. Opladen; Berlin [u.a.]: Budrich.

Plessner, Helmuth. 1976. Die Frage nach der Conditio humana, Frankfurt a. M.: Suhrkamp.

Simmel, Georg. 1922. Exkurs über das Problem: Wie ist Gesellschaft möglich. In Soziologie: Untersuchungen über die Formen der Vergesellschaftung, 21-30. München/Leipzig: Duncker und Humblot. 